\title{
Streptozotocin-Induced Diabetic Neuropathic Pain Is Associated with Potentiated Calcium-Permeable AMPA Receptor Activity in the Spinal Cord
}

\author{
Shao-Rui Chen, Jixiang Zhang, Hong Chen, and Hui-Lin Pan \\ Center for Neuroscience and Pain Research, Department of Anesthesiology and Perioperative Medicine, The University of Texas \\ MD Anderson Cancer Center, Houston, Texas
}

Received July 8, 2019; accepted August 20, 2019

\begin{abstract}
Neuronal hyperactivity in the spinal dorsal horn can amplify nociceptive input in diabetic neuropathic pain. The glutamate $\mathrm{N}$ methyl-D-aspartate and $\alpha$-amino-3-hydroxy-5-methyl-4-isoxazole propionic acid receptors (NMDA receptors and AMPA receptors, respectively) are involved in spinal nociceptive transmission. It is unclear, however, whether painful diabetic neuropathy is associated with changes in the activity of synaptic NMDA receptors and AMPA receptors in spinal dorsal horn neurons. AMPA receptors lacking GluA2 are $\mathrm{Ca}^{2+}$-permeable (CP-AMPA receptors), and their currents display characteristic inward rectification. In this study, we showed that evoked excitatory postsynaptic currents (EPSCs), induced by streptozotocin, exhibited inward rectification in spinal dorsal neurons in diabetic rats. Presynaptic and postsynaptic NMDA receptor activity in the spinal dorsal horn was similar in diabetic and control rats. In the dorsal spinal cord, the membrane GluA2 protein level was significantly lower in diabetic than in control rats, whereas the cytosolic GluA2 level was greater in diabetic than in control rats. In contrast, the GluA1 subunit levels in the plasma membrane and cytosol did not differ between the two groups. Blocking CPAMPA receptors significantly reduced the amplitude of EPSCs of
\end{abstract}

dorsal horn neurons in diabetic but not in control rats. Furthermore, blocking spinal CP-AMPA receptors reduced pain hypersensitivity in diabetic rats but had no effect on nociception in control rats. Our study suggests that diabetic neuropathy augments CP-AMPA receptor activity in the spinal dorsal horn by causing intracellular retention of GluA2 and impairing GluA2 membrane trafficking. Increased prevalence of spinal CP-AMPA receptors sustains diabetic neuropathic pain.

\section{SIGNIFICANCE STATEMENT}

This study demonstrates that the prevalence of synaptic calcium-permeable AMPA receptors is increased in the spinal dorsal horn, which mediates pain hypersensitivity in diabetic neuropathy. Thus, calcium-permeable AMPA receptors play an important role in glutamatergic synaptic plasticity in the spinal cord in painful diabetic neuropathy. This new knowledge improves our understanding of the mechanisms involved in central sensitization associated with diabetic neuropathic pain and suggests that calcium-permeable AMPA receptors are an alternative therapeutic target for treating this chronic pain condition.

\section{Introduction}

Peripheral neuropathy is a prevalent complication that afflicts people with a long history of diabetes. Chronic pain caused by diabetic neuropathy is not adequately relieved by available treatments and represents a major clinical problem. A decreased availability of various neurotrophic factors, mitochondrial dysfunction, and abnormal protein kinase $\mathrm{C}$ activation in sensory nerves may contribute to the development of diabetic neuropathy (Ahlgren and Levine, 1994; Tomlinson et al., 1997; Russell et al., 2002). Insulin deficiency is considered a major cause of sensory neuropathy in type 1 diabetes because early insulin therapy can normalize or impede the development of diabetic neuropathic pain in

This work was supported by a grant from the National Institutes of Health [R01 NS101880] and by the N.G. and Helen T. Hawkins Endowment.

https://doi.org/10.1124/jpet.119.261339. streptozotocin (STZ)-induced diabetic animals and in patients with diabetes (Sasaki et al., 1998; Brussee et al., 2004; Romanovsky et al., 2006; Hoybergs and Meert, 2007); however, the pathophysiological mechanism leading to chronic pain development in diabetic patients is not fully understood.

Spinal dorsal horn neurons are critically involved in processing sensory input and play a major role in the development of chronic neuropathic pain. Although painful diabetic neuropathy is associated with hyperactivity of spinal dorsal horn neurons (Chen and Pan, 2002), the underlying mechanisms remain unclear. In the spinal dorsal horn, glutamate, via acting on synaptic $\alpha$-amino-3-hydroxy-5methyl-4-isoxazolepropionic acid (AMPA) receptors and $\mathrm{N}$ methyl-D-aspartate (NMDA) receptors, is the most dominant excitatory neurotransmitter for the relay of nociceptive input. Previous work on targeting glutamate receptors for treating neuropathic pain has focused primarily on NMDA receptors because NMDA receptors contribute critically to

ABBREVIATIONS: AMPA, $\alpha$-amino-3-hydroxy-5-methyl-4-isoxazolepropionic acid; AP5, 2-amino-5-phosphonopentanoic acid; AMPAR, AMPA receptor; $\mathrm{CP}, \mathrm{Ca}^{2+}$-permeable; EPSC, excitatory postsynaptic current; mEPSC, miniature excitatory postsynaptic current; NMDA, N-methyl-Daspartate; STZ, streptozotocin. 
the development of chronic pain caused by traumatic nerve injury and chemotherapy (Chen et al., 2014b, 2018; Xie et al., $2016,2017)$; however, changes in the activity of presynaptic and postsynaptic NMDA receptors in the spinal cord in painful diabetic neuropathy remain unclear.

Similar to NMDA receptors, AMPA receptors (AMPARs) are crucially involved in various physiologic functions, such as learning and memory, and in the pathophysiology of many neurologic and psychologic conditions (Franciosi, 2001; Shepherd and Huganir, 2007; Traynelis et al., 2010). AMPARs are ion channels composed of a combination of GluA1, GluA2, GluA3, and GluA4 (Traynelis et al., 2010). At excitatory synapses, AMPARs contain predominantly heteromeric GluA1/ GluA2 subunits and, in some instances, of GluA3/GluA2 subunits (Kauer and Malenka, 2006; Derkach et al., 2007). GluA2 is highly significant for the biophysical properties of AMPARs because GluA2-containing AMPARs are impermeable to $\mathrm{Ca}^{2+}$. On the other hand, GluA2-lacking AMPARs show inward-rectifying currents and have a high $\mathrm{Ca}^{2+}$ permeability and are thus referred to as $\mathrm{Ca}^{2+}$-permeable AMPAs (CP-AMPARs) (Sommer et al., 1991; Chen et al., 2013). The prevalence of synaptic CP-AMPARs is markedly increased in the dorsal spinal cord after nerve ligation (Chen et al., 2013); however, it is unknown whether diabetic neuropathic pain is associated with increased activity of CP-AMPARs in the spinal cord. Therefore, in this study, we determined whether spinal NMDA receptors and AMPARs are differentially regulated in streptozotocin (STZ)-induced diabetic neuropathy.

\section{Materials and Methods}

Animal Model of Diabetic Neuropathic Pain. All experimental procedures were approved by the Animal Care and Use Committee of the University of Texas MD Anderson Cancer Center (approval no. 1186-RN01). Male Sprague-Dawley rats (8 weeks old; Harlan Sprague-Dawley, Indianapolis, IN) were used. Experimental diabetes was induced by intraperitoneal administration of $60 \mathrm{mg} / \mathrm{kg}$ of STZ, a pancreatic $\beta$-cell toxin (Chen and Pan, 2002; Chen et al., 2011). Age-matched saline-injected rats were used as the control group. This rat diabetic model mimics the clinical symptoms of diabetic patients and shows pain hypersensitivity and diminished opioid analgesic effects (Chen and Pan, 2002; Wang et al., 2007; Chen et al., 2011). Diabetes was confirmed by measuring the glucose concentration in the tail-vein blood samples using ACCU-CHEK test strips (Roche Diagnostics, Basel, Switzerland) 3 weeks after STZ injection. All animals were housed (two to three rats per cage) in a specific-pathogen-free facility and received standard chow and water ad libitum.

Intrathecal catheters were inserted in some rats anesthetized with inhalation of $2 \%$ to $3 \%$ isoflurane 2 weeks after diabetic induction. In brief, a small opening in the atlanto-occipital membrane was made, and a PE-10 catheter was introduced such that the caudal tip was situated at the L5-L6 spinal cord level (Chen et al., 2000). The animals were allowed to recover for at least 4 days before being used for intrathecal injections.

Assessment of Neuropathic Pain. To measure changes in the tactile sensitivity of the hind paw, rats were placed individually in chambers placed on a mesh floor. Calibrated von Frey filaments were applied vertically to the plantar surface of the hind paw to bend the filament for 6 seconds. A swift withdrawal was considered a positive response. The tactile stimulus producing a 50\% likelihood of withdrawal response was calculated by using the method described previously (Chen et al., 2000; Chen and Pan, 2002). To measure the mechanical nociceptive threshold, we applied a pressure stimulus on the hind paw using an analgesiometer (Ugo Basil, Varese, Italy). The device was activated to apply a constantly increasing force. When the animal displayed a paw withdrawal response, the device was instantly stopped to register the animal's withdrawal threshold (Chen and Pan, 2002; Chen et al., 2014a). The investigators testing the nociceptive behaviors were blinded to the treatments.

Spinal Cord Slice Preparation and Whole-Cell Recordings. All terminal electrophysiological experiments were performed on rats 3 weeks after STZ or vehicle treatment. Spinal cord slices at the L5-L6 levels were obtained from diabetic and control rats as we described previously (Li et al., 2002; Wang et al., 2007). We removed the lumbar spinal cord through laminectomy during isoflurane-induced anesthesia. We sliced the spinal cord to $400 \mu \mathrm{m}$ in thickness using a vibratome and continuously superfused the slices with artificial cerebrospinal fluid containing (in millimolars) $117.0 \mathrm{NaCl}, 2.5 \mathrm{CaCl}_{2}, 1.2 \mathrm{NaH}_{2} \mathrm{PO}_{4}$, $3.6 \mathrm{KCl}, 1.2 \mathrm{MgCl}_{2}, 11.0$ glucose, and $25.0 \mathrm{NaHCO}_{3}$. Neurons in the lamina II outer zone were chosen for recording because they mainly receive nociceptive input from primary afferent nerves (Pan and Pan, 2004; Wang et al., 2018). Whole-cell voltage clamp recordings were performed at $34^{\circ} \mathrm{C}$ by using pipettes filled with a solution containing (in millimolars) $110 \mathrm{Cs}_{2} \mathrm{SO}_{4}, 0.5 \mathrm{CaCl}_{2}, 5.0 \mathrm{HEPES}, 5.0$ EGTA, 5 TEA, $2.0 \mathrm{MgCl}_{2}, 5.0$ ATP-Mg, $0.5 \mathrm{Na}-\mathrm{GTP}, 0.1$ spermine, and 10 lidocaine $\mathrm{N}$-ethyl bromide. We included $0.1 \mathrm{mM}$ spermine in the intracellular recording solution to compensate for a possible loss of endogenous polyamines during whole-cell recording in spinal cord slices (Li et al., 2012; Chen et al., 2013).

The attached dorsal root was electrically stimulated $(0.6 \mathrm{~mA}, 0.2$ millisecond, and $0.1 \mathrm{~Hz}$ ) to evoke EPSCs. Monosynaptic EPSCs were identified when the evoked EPSCs showed a constant latency and no conduction failure in response to a $20-\mathrm{Hz}$ electrical stimulation, as we described previously (Li et al., 2002; Pan et al., 2002). Neurons were voltage-clamped at $-60 \mathrm{mV}$, and AMPAR-mediated excitatory postsynaptic currents (AMPARs-EPSCs) were recorded in the presence of $2 \mu \mathrm{M}$ strychnine, $10 \mu \mathrm{M}$ bicuculline, and $50 \mu \mathrm{M}$ DL-2-amino-5phosphonovaleric acid (AP5), a specific NMDA receptor antagonist (Chen et al., 2014a, 2018). GluA2-lacking CP-AMPARs exhibit inward rectification at positive holding potentials (Sommer et al., 1991; Bowie and Mayer, 1995; Chen et al., 2013). AMPAR-EPSCs were recorded at various membrane potentials ranging from -70 to $+70 \mathrm{mV}$ in $20-\mathrm{mV}$ steps to determine the current-voltage relationship. The evoked EPSCs are rapidly blocked by bath application of the AMPAR antagonist cyanquixaline (6-cyano-7-nitroquinoxaline-2,3-dione) ( $\mathrm{Li}$ et al., 2002; Pan et al., 2002).

Miniature EPSCs (mEPSCs) were recorded at a holding potential of $-60 \mathrm{mV}$ in the presence of $1 \mu \mathrm{M}$ strychnine, $10 \mu \mathrm{M}$ bicuculline, and $1 \mu \mathrm{M}$ tetrodotoxin. Presynaptic NMDA receptor-mediated glutamate release was tested by bath application of $50 \mu \mathrm{M}$ AP5 (Chen et al., 2014a, 2018), whereas postsynaptic NMDA receptor currents were elicited by puff application of $100 \mu \mathrm{M}$ NMDA to the recorded neuron using a positive-pressure system (4 p.s.i., 15 milliseconds; Toohey Company, Fairfield, NJ). To minimize the $\mathrm{Mg}^{2+}$ block of NMDA receptors, the puff NMDA currents were recorded in an extracellular solution containing no $\mathrm{Mg}^{2+}, 10 \mu \mathrm{M}$ glycine, and $1 \mu \mathrm{M}$ tetrodotoxin at a holding potential of $-60 \mathrm{mV}$ (Chen et al., 2014a, 2018).

AP5, cyanquixaline (6-cyano-7-nitroquinoxaline-2,3-dione), and bicuculline were obtained from Ascent Scientific (Princeton, NJ). IEM-1460 (N,N,H,-Trimethyl-5-[(tricyclo[3.3.1.13,7]dec-1-ylmethyl) amino]-1-pentanaminiumbromide hydrobromide) was purchased from Tocris Bioscience (Ellisville, MO). All drugs were prepared immediately before the experiments and delivered at the indicated final concentrations.

Immunoblotting. Plasma membrane and cytosolic proteins were isolated from the dorsal spinal cord at L5 and L6 levels using MemPER Plus Membrane Protein Extraction Kit (\#89842; Thermo Fisher Scientific, Waltham, MA). Tissues were added with $400 \mu$ l permeabilization buffer (no. 78440; Thermo Fisher Scientific) and homogenized with tissue grinder. After incubating for 10 minutes at $4^{\circ} \mathrm{C}$ with constant mixing, the homogenate was centrifuged at $16,000 \mathrm{~g}$ for 15 minutes at $4^{\circ} \mathrm{C}$. The supernatant containing cytosolic proteins was carefully transferred to a new tube. The pellet was resuspended in 
$400 \mu \mathrm{l}$ of solubilization buffer in the presence of a protease and phosphatase inhibitor cocktail and incubated for 30 minutes at $4^{\circ} \mathrm{C}$ with constant mixing. After centrifugation at $16,000 \mathrm{~g}$ for 15 minutes at $4^{\circ} \mathrm{C}$, the supernatant containing solubilized membrane and membrane-associated proteins was transferred to another new tube. A total of $40 \mu \mathrm{g}$ of protein was loaded and separated via electrophoresis using NuPAGE 4\%-12\% Bis-Tris Protein gels (cat. no. NP0321BOX; Invitrogen, Carlsbad, CA) and then transferred to a polyvinylidene difluoride membrane (cat. no. IPVH00010; Millipore, Burlington, MA) for immunoblotting. As we described previously (Chen et al., 2013), primary antibodies used were as follows: rabbit anti-GluA2 (1:1000 dilution, cat. no. AB10529; Millipore), rabbit antiGluA1 (1:1000 dilution, cat. no. AB1504; Millipore), mouse anti- $\beta$-actin (1:2000 dilution, cat. no. ab8226; Abcam, Cambridge, MA), and mouse anti- $\mathrm{Na}^{+}-\mathrm{K}^{+}$-ATPase (1:2000 dilution, cat. no. ab7671; Abcam). Horseradish peroxidase-conjugated anti-rabbit IgG (cat. no. 111-036-003, 1: 10,000; Jackson ImmunoResearch, West Grove, PA) and anti-mouse IgG (cat. no. 115-036-062, 1:10,000; Jackson ImmunoResearch) were used as secondary antibodies. The protein bands were detected using a chemiluminescence kit (cat. no. 34580; Thermo Fisher Scientific).
Data Analysis. Data are presented as mean \pm S.E.M. Only one neuron was recorded from each spinal cord slice, and at least five animals were used for each recording protocol. The mEPSCs were analyzed using a peak detection program (MiniAnalysis; Synaptosoft, Leonia, NJ). The amplitudes of evoked EPSCs were analyzed with Clampfit 9.2 (Molecular Devices, San Jose, CA). The rectification index was obtained by dividing the amplitude of AMPAR-EPSCs recorded at $+50 \mathrm{mV}$ by that at $-50 \mathrm{mV}$ (Li et al., 2012; Chen et al., 2013). Two-tailed Student's $t$ tests were used to compare two groups, and one-way analysis of variance (with Dunnett's post-hoc test) was used to determine the differences between more than two groups. All statistical analyses were performed using Prism software (version 7; GraphPad Software Inc., La Jolla, CA). $P$ value of less than 0.05 was considered statistically significant.

\section{Results}

Diabetic Neuropathy Does Not Change Spinal NMDA Receptor Activity at Presynaptic and Postsynaptic Sites. NMDA receptors expressed presynaptically in the
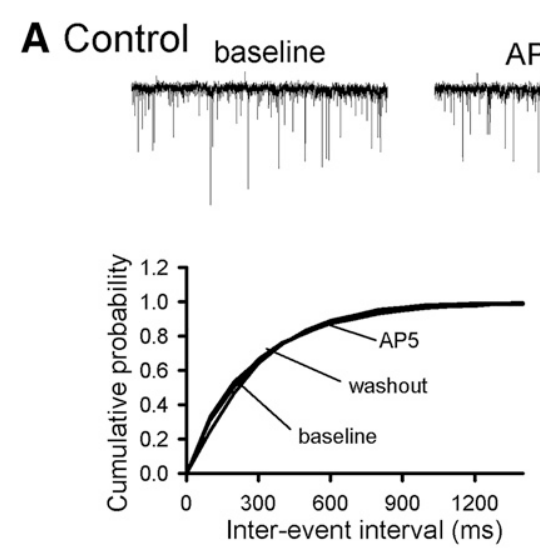

AP5
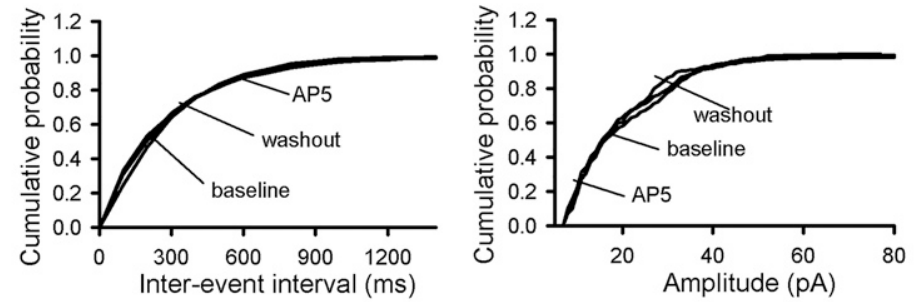

B Diabetic
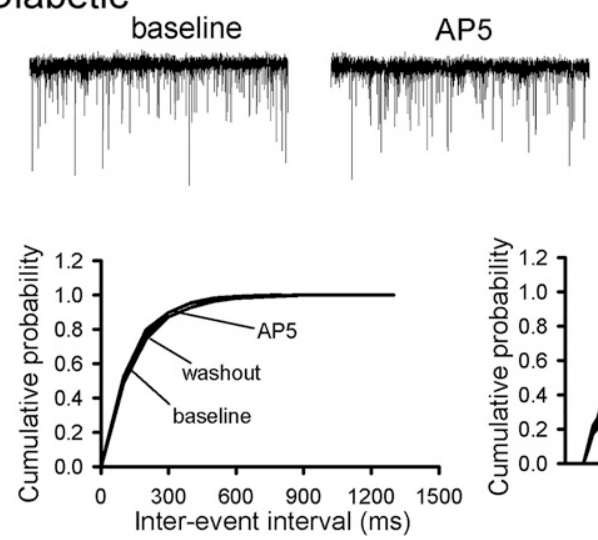

C

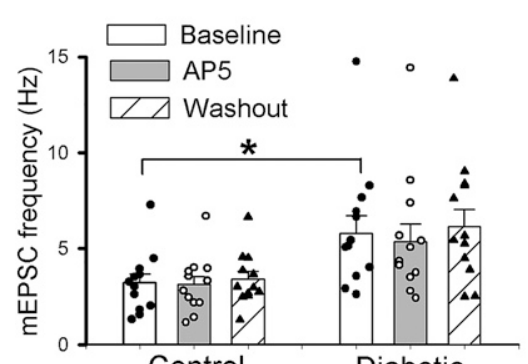

Control
Diabetic
Fig. 1. Painful diabetic neuropathy is not associated with changes in presynaptic NMDA receptor activity in the spinal dorsal horn. (A and B) Original recording traces and cumulative plots show the effect of $50 \mu \mathrm{M}$ AP5 on the frequency and amplitude of mEPSCs of lamina II neuron from control (A) and diabetic (B) rats. (C) Mean data show the effect of AP5 on the frequency and amplitude of mEPSCs in control and diabetic rats ( $n=12$ neurons in each group). Data are presented as means \pm S.E.M. $* P<0.05$, compared with the baseline in control rats (one-way ANOVA followed by Dunnett's post-hoc test).

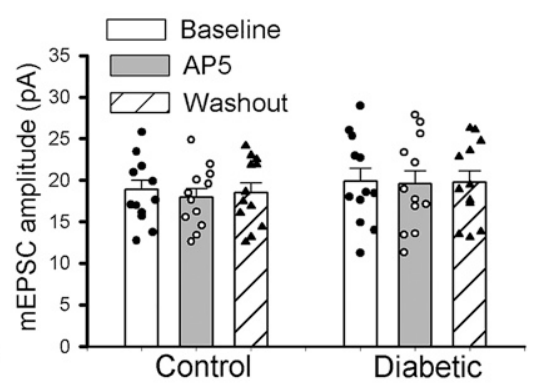

Control

Diabetic

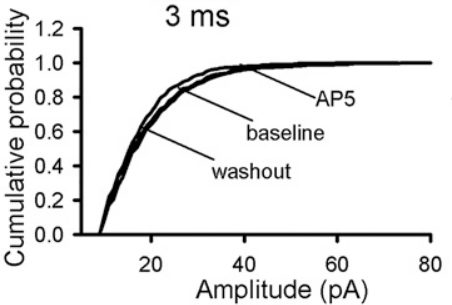

washout

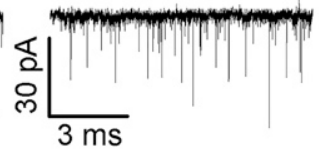

๕̊

washout

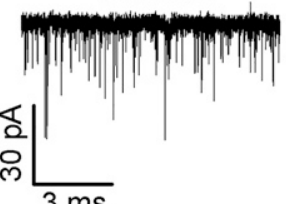


spinal cord are not activated under normal conditions but are spontaneously activated in neuropathic pain after peripheral nerve injury and treatment with calcineurin inhibitors and certain chemotherapeutics (Chen et al., 2014a,b, 2018; Xie et al., 2016, 2017; Deng et al., 2019). To determine whether painful diabetic neuropathy is associated with increased presynaptic NMDA receptor activity in the spinal dorsal horn, we first recorded mEPSCs of lamina II neurons, which represent glutamate release from presynaptic terminals (Chen et al., 2014a, 2018), and examined the effect of blocking NMDA receptors with AP5. As reported previously (Li et al., 2010), the baseline frequency of mEPSCs was significantly greater in diabetic than in vehicle-treated control rats $(P=0.03, F(5,66)=4.46 ; n=12$ neurons in each group; Fig. 1); however, bath application of $50 \mu \mathrm{M}$ of AP5 for 6 minutes had no significant effect on the frequency or amplitude of mEPSCs of lamina II neurons recorded from diabetic or control rats (Fig. 1).

Diabetic neuropathic pain is maintained by augmented sensory input from primary afferent nerves (Chen and Pan, 2002; Khan et al., 2002; Cao et al., 2011). We thus determined specifically whether diabetic neuropathic pain alters the activity of NMDA receptors expressed specifically at central terminals of primary sensory nerves in the spinal dorsal horn. We recorded EPSCs of lamina II neurons evoked monosynaptically from the dorsal root to assess NMDA receptor-mediated glutamate release (Zhou et al., 2010; Chen et al., 2014a, 2018). The amplitude of evoked EPSCs at baseline was significantly greater in the diabetic ( $n=13$ neurons) than in the control group ( $n=11$ neurons; $P=0.04, \mathrm{~F}(5,66)=3.99$; Fig. 2, A-C), which is in agreement with our previous reports (Wang et al., 2007; Li et al., 2010); however, bath application of $50 \mu \mathrm{M}$ of AP5 did not have any effect on evoked EPSCs in lamina II neurons tested from diabetic or control rats (Fig. 2, A-C).

We next studied whether diabetic neuropathic pain alters the activity of postsynaptic NMDA receptors in spinal dorsal horn neurons. We recorded NMDA receptor currents in lamina II neurons elicited by puff application of $100 \mu \mathrm{M}$ of NMDA (Chen et al., 2014a; Xie et al., 2017). The amplitude of puff NMDA-elicited currents in lamina II neurons between diabetic and control rats did not show any significant difference ( $n=11$ neurons in each group, Fig. 2, D and E). Collectively, these results suggest that painful diabetic neuropathy is not associated with altered activity of presynaptic or postsynaptic NMDA receptors in the spinal dorsal horn.

Activity of CP-AMPA Receptors in the Spinal Dorsal Horn Is Increased in Diabetic Neuropathy. Spinal AMPARs are critically involved in the relay of nociceptive information in traumatic nerve injury-induced neuropathic pain (Chen et al., 2000, 2013). To determine whether diabetic neuropathic pain alters the activity of GluA2-lacking, CPAMPAR $\$$ s in the spinal cord, we examined the current-voltage relationship of AMPARs-EPSCs of dorsal horn neurons in the presence of AP5. GluA2-lacking AMPARs are permeable to $\mathrm{Ca}^{2+}$ and display inward rectification at positive holding potentials owing to the voltage-dependent block by
A Control
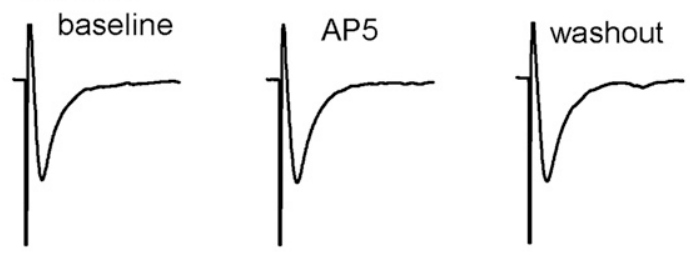

B Diabetic
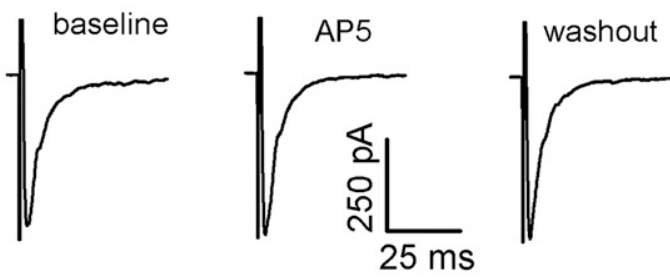

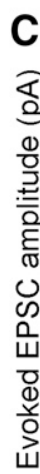

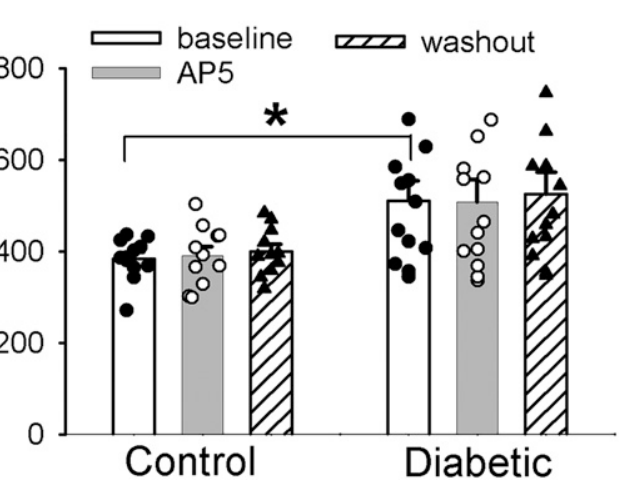

D

\section{Puff NMDA}
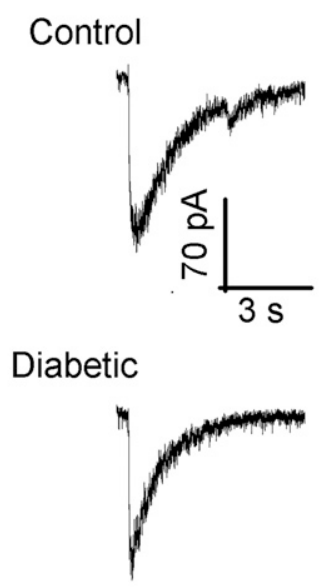

E

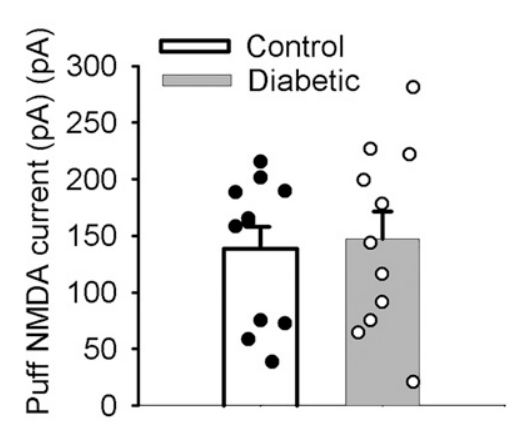

Fig. 2. Synaptic NMDA receptor activity in the spinal dorsal horn is not altered in painful diabetic neuropathy. (A-C) Original traces (A and B) and summary data $(\mathrm{C})$ show the effect of bath application of $50 \mu \mathrm{M}$ AP5 on evoked monosynaptic excitatory postsynaptic currents (EPSCs) of lamina II neurons from control ( $n=11$ neurons) and diabetic rats $(n=13$ neurons). ( $\mathrm{D}$ and $\mathrm{E}$ ) Representative traces (D) and mean data (E) show NMDA receptor currents elicited by puff application of $100 \mu \mathrm{M}$ NMDA onto lamina II neurons from control and diabetic rats $(n=11$ neurons in each group). Data are presented as means \pm S.E.M. ${ }^{*} P<0.05$ compared with baseline in control rats (one-way ANOVA followed by Dunnett's post-hoc test). 
intracellular polyamines. We therefore measured the currentvoltage relationship of AMPARs-EPSCs in lamina II neurons electrically evoked from the dorsal root at various holding potentials. The EPSCs recorded showed a linear currentvoltage relationship in all lamina II neurons from control rats ( $n=13$ neurons; Fig. 3, A and B), indicating that EPSCs are mediated mainly by GluA2-containing AMPARs. Remarkably, the amplitude of EPSCs in diabetic rats was reduced at positive holding potentials ( $n=14$ neurons; Fig. $3, \mathrm{~A}$ and $\mathrm{B}$ ). The rectification index $\left(\mathrm{I}_{+} 50 \mathrm{mv} / \mathrm{I}_{-50 \mathrm{mV}}\right)$ of AMPAR-EPSCs was significantly smaller in diabetic rats $(0.71 \pm 0.05, n=14$ neurons) than in control rats $(1.09 \pm 0.07, n=13$ neurons; $P=0.003, \mathrm{t}(12)=4.19$; Fig. $3 \mathrm{C})$.

To determine to what extent GluA2-lacking AMPARs contribute to the overall glutamatergic synaptic input to dorsal horn neurons, N,N,H,-trimethyl-5-[(tricyclo[3.3.1.13,7] dec-1-ylmethyl)amino]-1-pentanaminiumbromide hydrobromide (IEM-1460), a highly selective blocker of GluA2-lacking AMPARs (Samoilova et al., 1999; Rossi et al., 2008; Chen et al., 2013), was used. We tested the effect of IEM-1460 on monosynaptic AMPARs-EPSCs electrically evoked from the dorsal root at a holding potential at $-60 \mathrm{mV}$. IEM-1460 $(100 \mu \mathrm{M})$, via bath perfusion, did not affect the amplitude of EPSCs in dorsal horn neurons in control rats $(n=14$ neurons, Fig. 4). In contrast, IEM-1460 significantly attenuated the amplitude of evoked AMPARs-EPSCs in diabetic rats ( $n=13$ neurons, $F(5,75)=4.375, P=0.041$, Fig. 4). Together, these results suggest that painful diabetic neuropathy potentiates the activity of GluA2-lacking, CP-AMPARs in the spinal dorsal horn.

Diabetic Neuropathy Reduces Plasma Membrane Trafficking of GluA2 in the Spinal Cord. GluA1 and GluA2 subunits are the main AMPARs on the postsynaptic sites in the superficial spinal dorsal horn (Kerr et al., 1998). We therefore determined whether the protein amounts of spinal GluA1 and GluA2 subunits in the plasma membrane are altered in diabetic rats. Immunoblotting was used to measure the protein amount of GluA1 and GluA2 in the plasma membrane and cytosolic fractions. The protein amount of GluA2 in the plasma membrane in the diabetic group was significantly lower than that in the control group $(P=0.0017$, $t(12)=4.016, n=7$ rats per group; Fig. 5). Also, the GluA2 protein level in the cytosol in the diabetic rats was much greater than that in the control rats $(P=0.0011, t(12)=4.296$, $n=7$ rats per group; Fig. 5); however, the level of GluA1 in the plasma membrane and cytosol was similar in control and diabetic groups ( $n=7$ rats per group, Fig. 5 ). These results indicate that diabetic neuropathy causes intracellular GluA2 retention and attenuates GluA2 plasma membrane trafficking in the spinal dorsal horn.

Spinal CP-AMPA Receptors Mediate Pain Hypersensitivity Associated with Diabetic Neuropathy. We next determined whether CP-AMPARs at the spinal level are involved in maintaining chronic pain in STZ-induced diabetic rats. We tested the effect of IEM-1460 injected intrathecally on tactile allodynia and pressure hyperalgesia in diabetic rats. We did not test thermal sensitivity because the heat withdrawal threshold was similar in control and STZ-induced diabetic rats (Chen and Pan, 2002). Intrathecal administration of saline has no effect on the withdrawal thresholds tested with tactile and pressure stimuli in diabetic rats ( $\mathrm{Li}$ et al., 2010; Chen et al., 2011). In diabetic rats, intrathecal administration of IEM-1460 at 5-20 $\mu \mathrm{g}$ significantly elevated the tactile and pressure withdrawal thresholds in a dosedependent manner ( $n=7$ rats; Fig. 6 ). In contrast, intrathecal administration of $20 \mu \mathrm{g}$ of IEM-1460 did not significantly alter the tactile and pressure withdrawal thresholds in control rats ( $n=6$ rats; Fig. 6). These findings suggest that spinal CPAMPARs contribute to sustaining pain hypersensitivity associated with diabetic neuropathy.
A

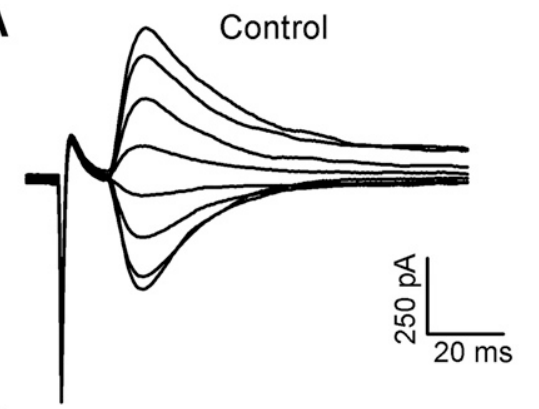

B

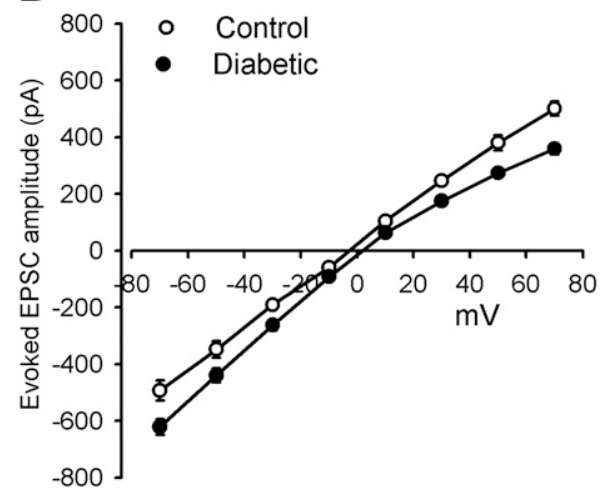

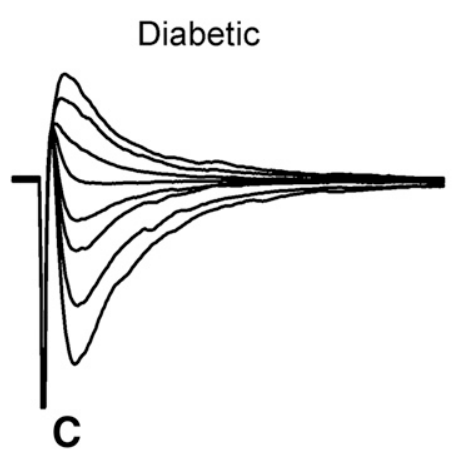

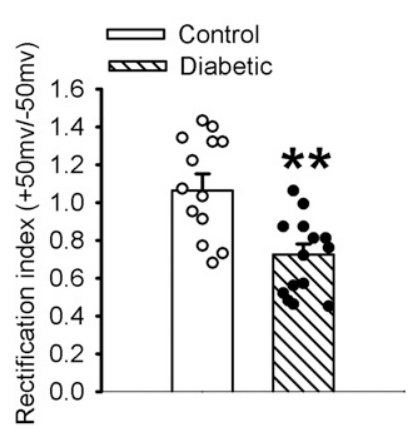

Fig. 3. Painful diabetic neuropathy increases CP-AMPA receptor activity of spinal dorsal horn neurons. (A) Representative recording traces of evoked EPSC of lamina II neurons (A) and their current-voltage curves (B) recorded at holding potentials from -70 to $+70 \mathrm{mV}$ in control ( $n=13$ neurons) and diabetic ( $n=14$ neurons) rats. (C) Mean data of the rectification index $\left(\mathrm{I}_{+50 \mathrm{mV}} / \mathrm{I}_{-50 \mathrm{mV}}\right)$ of evoked EPSCs of lamina II neurons in control $(n=13$ neurons) and diabetic ( $n=14$ neurons) rats. Data are presented as means \pm S.E.M. $* * P<0.01$, compared with control rats (two-tailed Student's $t$ test). 
A Control
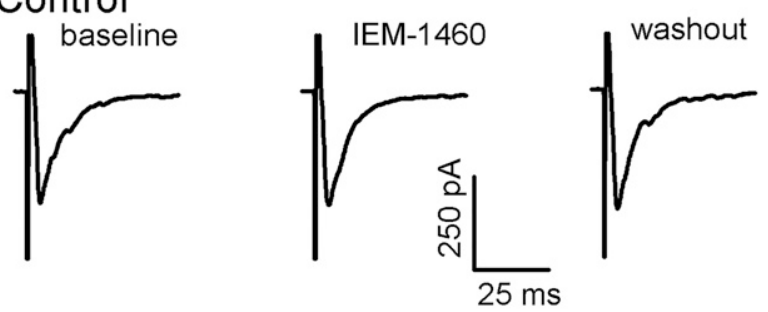

B Diabetic
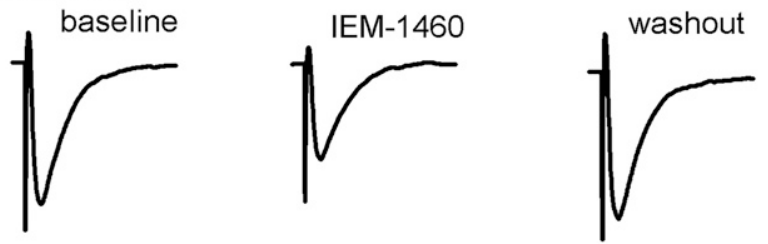

C
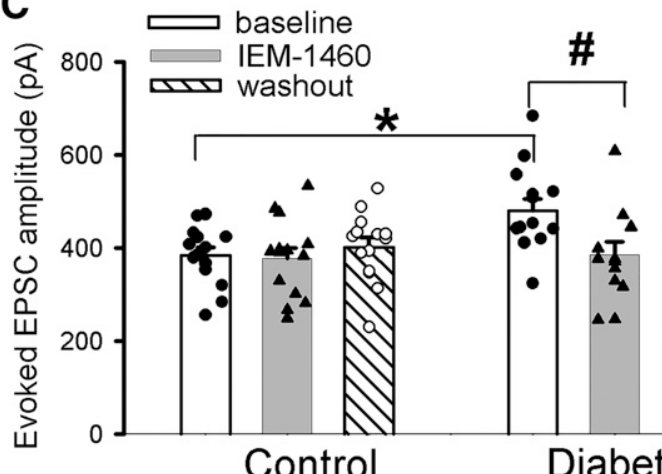

\section{Diabetic}

Fig. 4. CP-AMPA receptors contribute to increased glutamatergic input in the spinal dorsal horn in diabetic neuropathic pain. (A-C) Representative recording traces (A and $\mathrm{B}$ ) and summary data (C) show the differential effect of bath application of $100 \mu \mathrm{M}$ IEM-1460 on evoked EPSCs of lamina II neurons in control ( $n=14$ neurons) and diabetic rats ( $n=13$ neurons). The mean value in the control group was set at 1 . Data are presented as means \pm S.E.M. ${ }^{*} P<0.05$ compared with baseline in control rats. ${ }^{\#} P<0.05$ compared with the baseline in diabetic rats (oneway ANOVA followed by Dunnett's post-hoc test).

\section{Discussion}

Spinal cord NMDA receptors are generally thought to be central for neuropathic pain development (Zhou et al., 2011; Deng et al., 2019). For example, nerve ligation in rats potentiates the activity of both presynaptic and postsynaptic NMDA receptors in the spinal dorsal horn (Chen et al., 2014b, 2018). Furthermore, chemotherapy-induced neuropathic pain selectively potentiates presynaptic NMDA receptor activity of spinal dorsal horn neurons (Xie et al., 2016, 2017). In this study, however, we found that painful diabetic neuropathy induced by STZ is not associated with significant changes in the activity of presynaptic and postsynaptic NMDA receptors in the spinal dorsal horn. Thus, it is less likely that pain hypersensitivity in diabetic neuropathy results from altered NMDA receptor activity in the spinal dorsal horn. Although systemic treatment with an NMDA receptor blocker, meantime or MK801, for 1 to 2 weeks can reduce pain hypersensitivity in STZ-induced diabetic rats (Malcangio and Tomlinson, 1998; Daulhac et al., 2006; Chen et al., 2009), its site of action may be in the supraspinal region. Our findings reinforce the concept that the molecular mechanisms involved in neuropathic pain development are disease-specific and that mechanism-based treatments should be considered for different types of neuropathic pain conditions.

The most important finding of our study is the increased CP-AMPA receptor prevalence in the spinal dorsal horn synapses in diabetic neuropathic pain. Because AMPARs in the spinal dorsal horn are essential for synaptic and nociceptive transmission, determining the AMPAR-associated synaptic plasticity in neuropathic pain is fundamentally important. GluA2 acts in a dominant fashion to control several receptor properties including $\mathrm{Ca}^{2+}$ permeability and block by intracellular polyamines. Most AMPARs in the brain and spinal cord contain GluA2, which makes AMPARs $\mathrm{Ca}^{2+}$. impermeable (Dingledine et al., 1999; Isaac et al., 2007). This is crucial for maintaining a low level of cytoplasmic $\mathrm{Ca}^{2+}$ under physiologic conditions. Thus, alterations in GluA2 composition could have a dramatic impact on the quality and strength of synaptic transmission (Plant et al., 2006; Derkach et al., 2007; Li et al., 2012). GluA2-deficient mice display facilitated nociceptive plasticity and long-lasting pain hypersensitivity after tissue inflammation (Hartmann et al., 2004). We found that diabetic neuropathic pain remarkably changed the current-voltage relationship of AMPARs from linear to inward-rectifying, suggesting a change from GluA2containing AMPARs to GluA2-lacking CP-AMPARs at synaptic sites in the spinal dorsal horn. A persistent incorporation of CP-AMPARs in neurons could cause excessive $\mathrm{Ca}^{2+}$ influx (Kwak and Weiss, 2006), leading to central sensitization in diabetic neuropathy (Chen and Pan, 2002).

The exact molecular mechanisms responsible for the augmented activity of CP-AMPARs in the spinal cord in painful diabetic neuropathy remain unknown. In neuropathic pain caused by traumatic nerve injury, increased NMDA receptor activity seems to play a main role in the switch to CP-AMPARs in spinal dorsal horn neurons (Chen et al., 2013); however, we found no evidence of increased NMDAR activity in the spinal cord in STZ-induced diabetic rats. CP-AMPARs are present in spinal dorsal horn neurons (Engelman et al., 1999; Youn et al., 2008). It is likely that some CP-AMPARs are present in the extrasynaptic site and can substitute postsynaptic GluA2containing AMPARs in diabetic neuropathy. We showed that in the spinal cord of diabetic rats, the GluA2 protein level was reduced in the plasma membranes but was increased in the cytosol. Thus, augmented GluA2 internalization and reduced GluA2 membrane trafficking in the spinal dorsal horn can reduce the amount of GluA2-containing $\left(\mathrm{Ca}^{2+}\right.$-impermeable) AMPARs and increases the prevalence and activity of GluA2lacking, CP-AMPARs at postsynaptic sites in painful diabetic neuropathy.

We showed that the activity of CP-AMPARs is increased and mediates the augmented nociceptive input to spinal dorsal horn neurons in STZ-induced diabetic neuropathy. Reducing the activity of AMPARs, particularly CP-AMPARs, at the spinal cord level effectively relieves neuropathic pain induced by traumatic nerve injury (Chen et al., 2000, 2013). In this study, we found that IEM-1460, a selective CP-AMPAR blocker (Samoilova et al., 1999; Kobylecki et al., 2010), preferentially reduced the glutamatergic EPSCs of dorsal horn neurons in diabetic but not in control rats. Furthermore, we found that blocking CP-AMPARs at the spinal cord level markedly attenuated pain hypersensitivity in STZ-induced diabetic rats but produced no effect on the nociceptive threshold in control rats. These data suggest that in the spinal 


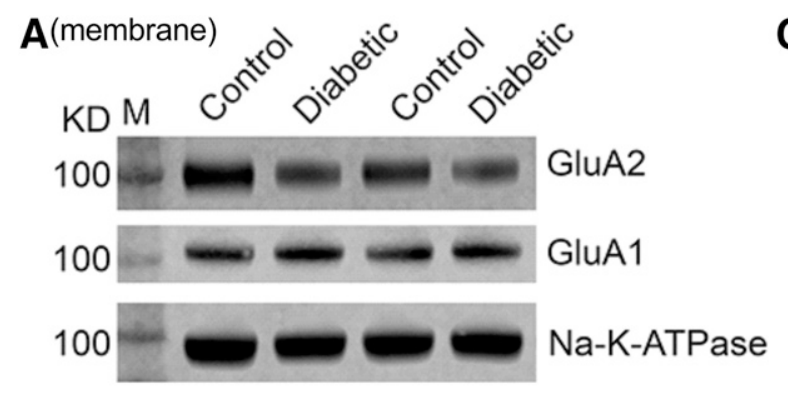

B

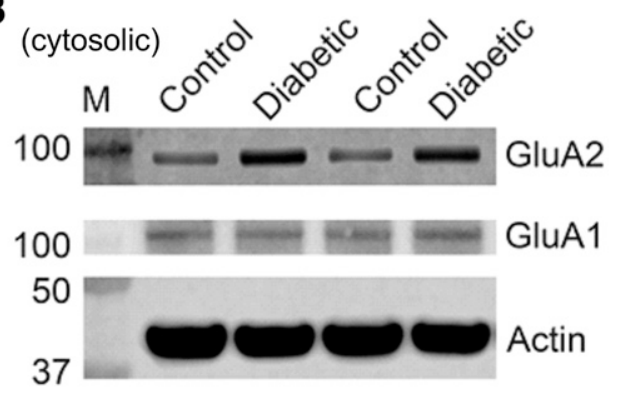

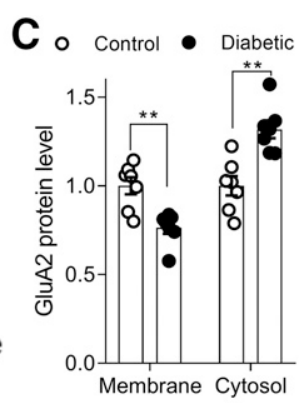

D

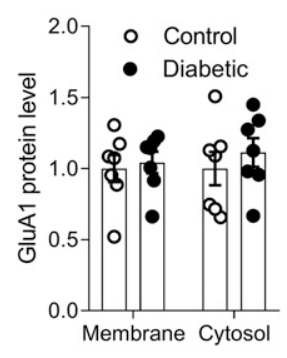

Fig. 5. The plasma membrane trafficking of GluA2 is reduced in the dorsal spinal cord of diabetic rats. (A and $\mathrm{B}$ ) Representative blotting images show the protein level of GluA1 and GluA2 in the plasma membrane (A) and cytosol (B) in the dorsal spinal cords between diabetic rats and control groups. $\mathrm{Na}^{+}-\mathrm{K}^{+}$-ATPase and $\beta$-actin were used as loading controls for plasma membrane and cytosolic proteins, respectively. M, molecular weight marker. (C and D) Group data show the protein level of GluA2 (C) and GluA1 (D) in the plasma membrane and cytosolic fractions of the dorsal spinal cord tissues in control and diabetic rats. Data are shown as means \pm S.E.M., $n=7$ rats in each group. $* * P<0.01$ compared with the control group (twotailed Student's $t$ test). dorsal horn, increased activity of CP-AMPARs may serve to strengthen nociceptive transmission in painful diabetic neuropathy. We showed that the amplitude of glutamatergic EPSCs evoked from the dorsal root is significantly larger in diabetic than in control rats (Wang et al., 2007; Li et al., 2010). The increased nociceptive glutamatergic input from primary sensory neurons to the spinal dorsal horn in painful diabetic neuropathy is caused by reduced $\mathrm{K}^{+}$channel activity and/or
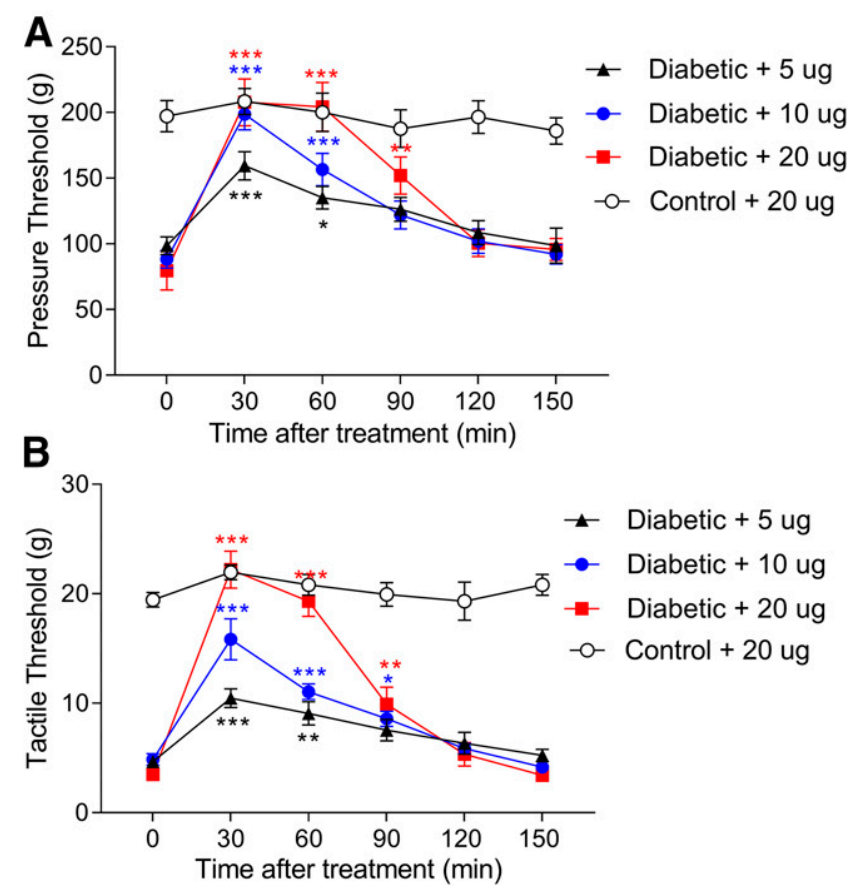

Fig. 6. CP-AMPA receptors at the spinal cord level contribute to diabetic neuropathic pain. (A and B) Time course of the inhibitory effect of of IEM1460 injected intrathecally on the withdrawal threshold in response to a noxious pressure stimulus (A) and von Frey filaments (B) in seven diabetic rats and six control rats. Data are shown as means \pm S.E.M. $* P<0.05 ; * * P<0.01 ; * * * P<0.001$ compared with respective baseline values (time 0; repeated measures ANOVA with Dunnett's post-hoc test). increased activity of voltage-activated $\mathrm{Ca}^{2+}$ channels and mGluR5 in dorsal root ganglion neurons (Hall et al., 2001; Cao et al., 2010, 2011; Li et al., 2010).

In conclusion, by using electrophysiological, biochemical, and behavioral approaches, we provide new evidence that the prevalence of synaptic CP-AMPARs is increased in the spinal dorsal horn, which mediates pain hypersensitivity in diabetic neuropathy. Thus, CP-AMPARs play a key role in glutamatergic synaptic plasticity in the spinal cord in painful diabetic neuropathy. This new information advances our knowledge of the underlying mechanisms responsible for synaptic plasticity in diabetic neuropathic pain and suggests that CP-AMPARs are an alternative therapeutic target for treating this chronic pain condition.

\section{Authorship Contributions}

Participated in research design: S.-R. Chen, Pan.

Conducted experiments: S.-R. Chen, Zhang, H. Chen.

Performed data analysis: S.-R. Chen, Zhang, H. Chen.

Wrote or contributed to the writing of the manuscript: S.-R.Chen, Pan.

\section{References}

Ahlgren SC and Levine JD (1994) Protein kinase C inhibitors decrease hyperalgesia and C-fiber hyperexcitability in the streptozotocin-diabetic rat. J Neurophysiol 72: 684-692.

Bowie D and Mayer ML (1995) Inward rectification of both AMPA and kainate subtype glutamate receptors generated by polyamine-mediated ion channel block. Neuron 15:453-462.

Brussee V, Cunningham FA, and Zochodne DW (2004) Direct insulin signaling of neurons reverses diabetic neuropathy. Diabetes 53:1824-1830.

Cao XH, Byun HS, Chen SR, Cai YQ, and Pan HL (2010) Reduction in voltagegated $\mathrm{K}+$ channel activity in primary sensory neurons in painful diabetic neuropathy: role of brain-derived neurotrophic factor. J Neurochem 114: 1460-1475.

Cao XH, Byun HS, Chen SR, and Pan HL (2011) Diabetic neuropathy enhances voltage-activated $\mathrm{Ca} 2+$ channel activity and its control by M4 muscarinic receptors in primary sensory neurons. $J$ Neurochem 119:594-603.

Chen J, Li L, Chen SR, Chen H, Xie JD, Sirrieh RE, MacLean DM, Zhang Y, Zhou $\mathrm{MH}$, Jayaraman V, et al. (2018) The $\alpha 2 \delta-1-N M D A$ receptor complex is critically involved in neuropathic pain development and gabapentin therapeutic actions. Cell Rep 22:2307-2321.

Chen SR, Chen H, Yuan WX, and Pan HL (2011) Increased presynaptic and postsynaptic $\alpha 2$-adrenoceptor activity in the spinal dorsal horn in painful diabetic neuropathy. J Pharmacol Exp Ther 337:285-292.

Chen SR, Eisenach JC, McCaslin PP, and Pan HL (2000) Synergistic effect between intrathecal non-NMDA antagonist and gabapentin on allodynia induced by spinal nerve ligation in rats. Anesthesiology 92:500-506. 
Chen SR, Hu YM, Chen H, and Pan HL (2014a) Calcineurin inhibitor induces pain hypersensitivity by potentiating pre- and postsynaptic NMDA receptor activity in spinal cords. J Physiol 592:215-227.

Chen SR and Pan HL (2002) Hypersensitivity of spinothalamic tract neurons associated with diabetic neuropathic pain in rats. J Neurophysiol 87:2726-2733.

Chen SR, Samoriski G, and Pan HL (2009) Antinociceptive effects of chronic administration of uncompetitive NMDA receptor antagonists in a rat model of diabetic neuropathic pain. Neuropharmacology 57:121-126.

Chen SR, Zhou HY, Byun HS, Chen H, and Pan HL (2014b) Casein kinase II regulates N-methyl-D-aspartate receptor activity in spinal cords and pain hypersensitivity induced by nerve injury. J Pharmacol Exp Ther 350:301-312.

Chen SR, Zhou HY, Byun HS, and Pan HL (2013) Nerve injury increases GluA2 lacking AMPA receptor prevalence in spinal cords: functional significance and signaling mechanisms. J Pharmacol Exp Ther 347:765-772.

Daulhac L, Mallet C, Courteix C, Etienne M, Duroux E, Privat AM, Eschalier A and Fialip J (2006) Diabetes-induced mechanical hyperalgesia involves spinal mitogen-activated protein kinase activation in neurons and microglia via N-methyl-D-aspartate-dependent mechanisms. Mol Pharmacol 70:1246-1254.

Deng M, Chen SR, and Pan HL (2019) Presynaptic NMDA receptors control nociceptive transmission at the spinal cord level in neuropathic pain. Cell Mol Life Sci 76:1889-1899.

Derkach VA, Oh MC, Guire ES, and Soderling TR (2007) Regulatory mechanisms of AMPA receptors in synaptic plasticity. Nat Rev Neurosci 8:101-113.

Dingledine R, Borges K, Bowie D, and Traynelis SF (1999) The glutamate receptor ion channels. Pharmacol Rev 51:7-61.

Engelman HS, Allen TB, and MacDermott AB (1999) The distribution of neurons expressing calcium-permeable AMPA receptors in the superficial laminae of the spinal cord dorsal horn. J Neurosci 19:2081-2089.

Franciosi S (2001) AMPA receptors: potential implications in development and disease. Cell Mol Life Sci 58:921-930.

Hall KE, Liu J, Sima AA, and Wiley JW (2001) Impaired inhibitory G-protein function contributes to increased calcium currents in rats with diabetic neuropathy. $J$ Neurophysiol 86:760-770.

Hartmann B, Ahmadi S, Heppenstall PA, Lewin GR, Schott C, Borchardt T, Seeburg PH, Zeilhofer HU, Sprengel R, and Kuner R (2004) The AMPA receptor subunits GluR-A and GluR-B reciprocally modulate spinal synaptic plasticity and in flammatory pain. Neuron 44:637-650.

Hoybergs YM and Meert TF (2007) The effect of low-dose insulin on mechanical sensitivity and allodynia in type I diabetes neuropathy. Neurosci Lett 417:149-154.

Isaac JT, Ashby MC, and McBain CJ (2007) The role of the GluR2 subunit in AMPA receptor function and synaptic plasticity. Neuron 54:859-871.

Kauer JA and Malenka RC (2006) LTP: AMPA receptors trading places. Nat Neurosci 9:593-594.

Kerr RC, Maxwell DJ, and Todd AJ (1998) GluR1 and GluR2/3 subunits of the AMPA-type glutamate receptor are associated with particular types of neurone in laminae I-III of the spinal dorsal horn of the rat. Eur J Neurosci 10:324-333.

Khan GM, Chen SR, and Pan HL (2002) Role of primary afferent nerves in allodynia caused by diabetic neuropathy in rats. Neuroscience 114:291-299.

Kobylecki C, Cenci MA, Crossman AR, and Ravenscroft P (2010) Calcium-permeable AMPA receptors are involved in the induction and expression of l-DOPA-induced dyskinesia in Parkinson's disease. J Neurochem 114:499-511.

Kwak S and Weiss JH (2006) Calcium-permeable AMPA channels in neurodegenerative disease and ischemia. Curr Opin Neurobiol 16:281-287.

Li DP, Byan HS, and Pan HL (2012) Switch to glutamate receptor 2-lacking AMPA receptors increases neuronal excitability in hypothalamus and sympathetic drive in hypertension. $J$ Neurosci 32:372-380.

Li DP, Chen SR, Pan YZ, Levey AI, and Pan HL (2002) Role of presynaptic muscarinic and $\mathrm{GABA}(\mathrm{B})$ receptors in spinal glutamate release and cholinergic analgesia in rats. J Physiol 543:807-818.

Li JQ, Chen SR, Chen H, Cai YQ, and Pan HL (2010) Regulation of increased glutamatergic input to spinal dorsal horn neurons by mGluR5 in diabetic neuropathic pain. $J$ Neurochem 112:162-172.

Malcangio M and Tomlinson DR (1998) A pharmacologic analysis of mechanical hyperalgesia in streptozotocin/diabetic rats. Pain $\mathbf{7 6}: 151-157$.
Pan YZ, Li DP, and Pan HL (2002) Inhibition of glutamatergic synaptic input to spinal lamina $\mathrm{II}(\mathrm{o})$ neurons by presynaptic alpha(2)-adrenergic receptors. J Neurophysiol 87:1938-1947.

Pan YZ and Pan HL (2004) Primary afferent stimulation differentially potentiates excitatory and inhibitory inputs to spinal lamina II outer and inner neurons. $J$ Neurophysiol 91:2413-2421.

Plant K, Pelkey KA, Bortolotto ZA, Morita D, Terashima A, McBain C.J, Collingridge GL, and Isaac JT (2006) Transient incorporation of native GluR2-lacking AMPA receptors during hippocampal long-term potentiation. Nat Neurosci $\mathbf{9}$ 602-604.

Romanovsky D, Cruz NF, Dienel GA, and Dobretsov M (2006) Mechanical hyperalgesia correlates with insulin deficiency in normoglycemic streptozotocin-treated rats. Neurobiol Dis 24:384-394.

Rossi B, Maton G, and Collin T (2008) Calcium-permeable presynaptic AMPA receptors in cerebellar molecular layer interneurones. $J$ Physiol 586:5129-5145.

Russell JW, Golovoy D, Vincent AM, Mahendru P, Olzmann JA, Mentzer A, and Feldman EL (2002) High glucose-induced oxidative stress and mitochondrial dysfunction in neurons. FASEB $J$ 16:1738-1748.

Samoilova MV, Buldakova SL, Vorobjev VS, Sharonova IN, and Magazanik LG (1999) The open channel blocking drug, IEM-1460, reveals functionally distinct alpha-amino-3-hydroxy-5-methyl-4-isoxazolepropionate receptors in rat brain neurons. Neuroscience 94:261-268.

Sasaki T, Yasuda H, Maeda K, and Kikkawa R (1998) Hyperalgesia and decreased neuronal nitric oxide synthase in diabetic rats. Neuroreport 9:243-247.

Shepherd JD and Huganir RL (2007) The cell biology of synaptic plasticity: AMPA receptor trafficking. Annu Rev Cell Dev Biol 23:613-643.

Sommer B, Köhler M, Sprengel R, and Seeburg PH (1991) RNA editing in brain controls a determinant of ion flow in glutamate-gated channels. Cell 67:11-19.

Tomlinson DR, Fernyhough P, and Diemel LT (1997) Role of neurotrophins in diabetic neuropathy and treatment with nerve growth factors. Diabetes $\mathbf{4 6}$ (Suppl 2): S43-S49.

Traynelis SF, Wollmuth LP, McBain CJ, Menniti FS, Vance KM, Ogden KK, Hansen KB, Yuan H, Myers SJ, and Dingledine R (2010) Glutamate receptor ion channels: structure, regulation, and function. Pharmacol Rev 62:405-496.

Wang L, Chen SR, Ma H, Chen H, Hittelman WN, and Pan HL (2018) Regulating nociceptive transmission by VGluT2-expressing spinal dorsal horn neurons. $J$ Neurochem 147:526-540.

Wang XL, Zhang HM, Chen SR, and Pan HL (2007) Altered synaptic input and GABAB receptor function in spinal superficial dorsal horn neurons in rats with diabetic neuropathy. J Physiol 579:849-861.

Xie JD, Chen SR, Chen H, and Pan HL (2017) Bortezomib induces neuropathic pain through protein kinase C-mediated activation of presynaptic NMDA receptors in the spinal cord. Neuropharmacology 123:477-487.

Xie JD, Chen SR, Chen H, Zeng WA, and Pan HL (2016) Presynaptic N-methyl-daspartate (NMDA) receptor activity is increased through protein kinase $\mathrm{C}$ in paclitaxel-induced neuropathic pain. J Biol Chem 291:19364-19373.

Youn DH, Royle G, Kolaj M, Vissel B, and Randić M (2008) Enhanced LTP of primary afferent neurotransmission in AMPA receptor GluR2-deficient mice. Pain 136: 158-167.

Zhou HY, Chen SR, Chen H, and Pan HL (2010) Opioid-induced long-term potentiation in the spinal cord is a presynaptic event. J Neurosci 30:4460-4466.

Zhou HY, Chen SR, and Pan HL (2011) Targeting N-methyl-D-aspartate receptors for treatment of neuropathic pain. Expert Rev Clin Pharmacol 4:379-388.

Address correspondence to: Dr. Hui-Lin Pan, Department of Anesthesiology and Perioperative Medicine, The University of Texas MD Anderson Cancer Center, 1515 Holcombe Blvd., Unit 110, Houston, TX 77030. E-mail: huilinpan@mdanderson.org; or Dr. Shao-Rui Chen, Department of Anesthesiology and Perioperative Medicine, The University of Texas MD Anderson Cancer Center, 1515 Holcombe Blvd., Unit 110, Houston, TX 77030. E-mail: schen@mdanderson.org 